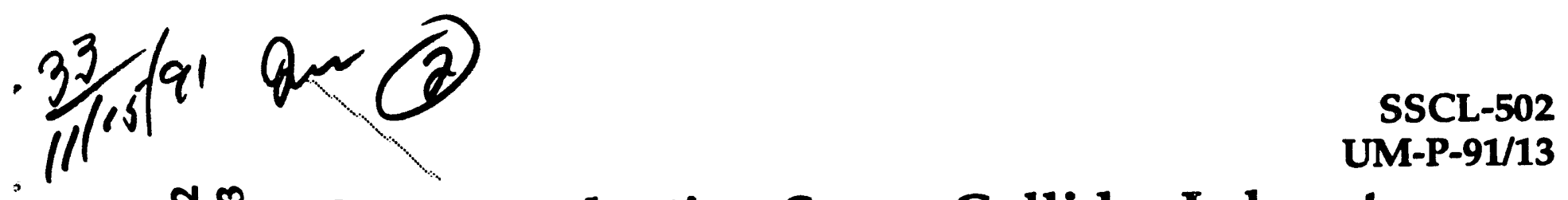

嘢 Superconducting Super Collider Laboratory

幽蓄

\title{
Quantum Electrodynamics With Complex Fermion Mass
}

B. McKellar and Dan-di Wu

August 1991 


\title{
Quantum Electrodynamics With Complex Fermion Mass*
}

\author{
Bruce J.H. McKellar ${ }^{1}$ and Dan-di $\mathrm{Wu}^{1,2,3}$ \\ ${ }^{1}$ School of Physics \\ University of Melbourne \\ Parkville, Victoria, 3052 \\ Australia \\ ${ }^{2}$ Institute of High Energy Physics \\ Beijing 100039 \\ P.R. China \\ ${ }^{3}$ Present Address: \\ Superconducting Super Collider Laboratory ${ }^{\dagger}$ \\ 2550 Beckleymeade Avenue \\ Dallas, Texas 75237 \\ USA \\ E-mail: DANWU@SSCVX1.SSC.GOV
}

August 1991

\section{DISCLAIMER}

\begin{abstract}
This report was prepared as an account of work sponsored by an agency of the United States Government. Neither the United States Government nor any agency thereof, nor any of their

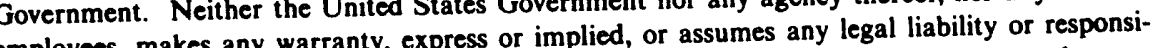
employees, makes any warranty, express or implied, or assumes any legal hability or respot, bility for the accuracy, completeness, or usefulness of any information, apparatus, product, or process disclosed, or represents that its use would not infringe privale by trade name, trademark, ence herein to any specific commercial product, process, or service by trade name, trademark, manufacturer, or otherwise does not necessarily constitute or imply its cindorsement, recommendation, or favoring by the United States Government or any agency thereof. and opinions of authors expressed herein do not necessarily state or reflect those of the United States Government or any agency thereof.
\end{abstract}

* This work is supported in part by the Australia Research Council.

$\dagger$ Operated by the Universities Research Association, Inc., for the U.S. Department of Energy under Contract No. DE-AC35-89ER40486. 
SSCL-502

UM-P-91/13

\title{
Quantum Electrodynamics With Complex Fermion Mass
}

\author{
Bruce J.H. McKellar and Dan-di Wu
}

\begin{abstract}
The quantum electrodynamics (QED) with a complex fermion mass - that is, a fermion mass with a chiral phase-is restudied, together with its chirally rotated version. We show how fake electric dipole moment can be obtained and how to avoid it.
\end{abstract}


Complex fermion mass or fermion mass with a chiral phase is allowed in quantum electrodynamics (QED) beci $\mathrm{i}$ e the phase has no effects, as people commonly believe. Please note that what we talk about is not the effective complex mass of a decaying particle. We are talking about the most general fermion mass term in fundamental theories. Complex fermion mass in QED has been studied by Fienberg, Kabir, and Weinberg (FKW). ${ }^{1}$ Interest in complex mass has been renewed in the context of the strong interaction by Dashen, ${ }^{2}$ and in the context of the strong CP by Peccei and Quinn ${ }^{3}$ and by Baluni and others. ${ }^{4}$ Recently some controversial ideas about strong CP have been raised by a few authors. ${ }^{5}$ Although the phase of the complex mass is redundant in QED, it may sometimes produce a fake electric dipole moment (EDM) and cause confusion. Indeed there are different explanations of why the phase of the fermion mass does not have an effect; according to some of these explanations, the same EDM can be identified as a reality if there are interactions other than electromagnetism. With this uncomfortable situation we feel it might be useful first to obtain a better understanding of why EDM does not appear in QED, and in particular how fake EDM is produced and how to eliminate it. Of course, QED is much simpler than QCD (quantum chromodynamics) in this context ${ }^{6}$ because at least people believe there is no condensate in QED. This is why we pick up QED; a conclusion in QED should be very clean.

A complex mass is

$$
\tilde{m}=m e^{-i \gamma_{5} \phi}=m \cos \phi-i m \gamma_{5} \sin \phi \equiv m_{1}-i \gamma_{5} m_{2}
$$

where $\phi$ is an arbitrary phase parameter with the periodicity $2 \pi$. The Lagrangian of interest is

$$
\mathcal{L}=-1 / 4 F^{\mu \nu} F_{\mu \nu}+i \bar{\psi}(\not \partial+i e \not 1) \psi-\bar{\psi} \tilde{m} \psi
$$

The only difference between this Lagrangian and the standard QED Lagrangian is the appearance of the parameter $\phi$. It seems that Lagrangian Eq. (2) breaks the standard space reflection $(\mathrm{P})$ and time reversal $(\mathrm{T})$ invariance while it conserves the charge conjugation (C) due to the complex mass. If this phase has any effects, we have the problem of why these effects are not seen, because there are no theoretical reasons to restrict $\phi$ to vanish. However, the question of whether $\mathrm{P}$ - and T-violating effects appear in the system is quite subtle and sometimes confusing, although the conclusion is clear as FKW claimed. The reason for this subtlety will gradually emerge in our later discussions, together with the correct treatment. Our basic approach will be to work directly with $\mathcal{L}$ in (2). 
One of the approaches is related to the study of the chiral-transformed version of $\mathcal{L}$ $\left(\mathcal{L}^{\prime}\right)$, which has a real fermion mass term. The required chiral transformation is

$$
\psi \longrightarrow \psi^{\prime}=e^{-i \gamma_{s} \phi / 2} \psi
$$

and $\mathcal{L}^{\prime}$ is

$$
\mathcal{L}^{\prime}=-1 / 4 F^{\prime \mu \nu} F_{\mu \nu}^{\prime}+1 / 4 \theta F^{\prime \mu \nu} \tilde{F}_{\mu \nu}^{\prime}+i \bar{\psi}^{\prime}\left(\not \partial+i e \not A^{\prime}\right) \psi^{\prime}-\bar{\psi}^{\prime} m \psi^{\prime}
$$

where $\left(\alpha=e^{2} / 4 \pi\right)$

$$
\tilde{F} \mu \nu=1 / 2 \varepsilon_{\mu \nu \varrho \sigma} F^{\varrho \sigma}, \quad \theta=\alpha \phi / 2 \pi .
$$

The $\theta$ of interest here satisfies $|\theta| \leq \alpha \ll 1$. The $\theta$ term (the second one) is due to the triangle anomaly. ${ }^{7}$ We have been careful here to allow the possibility that $A_{\mu}^{\prime}\left(F_{\mu \nu}^{\prime}\right)$ after chiral rotation will differ from $A_{\mu}\left(F_{\mu \nu}\right)$ before chiral rotation. Note the difference of $\mathcal{L}^{\prime}$ from $\mathcal{L}$ : the appearance of the $\theta$ term in addition to the change of the fermion mass term. These differences clearly pronounce that chiral rotation is not the symmetry of the system. Making chiral rotation is akin to transforming from an inertial frame of system to an accelerative one. Some aspects of the physical laws may change, unless special care is taken to compensate the change of Lagrangian by corresponding change of the wave functions, including the vacuum wave function. Therefore, the property of $\mathcal{L}^{\prime}$ in terms of $\mathrm{P}$ and $\mathrm{T}$ symmetries may not tell us much about Lagrangian $\mathcal{L}$.

Some previous authors throw away the $\theta$ term in Eq. (4) by saying that $F \tilde{F}$ is a complete divergence of some 4-vector, $F \tilde{F}=\partial^{\mu} K_{\mu}$. In this way, these authors conclude that $\mathcal{L}$ is $\mathrm{P}$ - and T-symmetric, as is $\mathcal{L}^{\prime}$, because they believe $\mathcal{L}$ and $\mathcal{L}^{\prime}$ describe the same system equivalently. This may or may not also pose questions. Indeed, a term which is a complete derivative with respect to time in the Lagrangian will not contribute in the Euler-Lagrangian equation, but it may contribute if it be otherwise. The fields in the Lagrangian are allowed to be unphysical in general. Therefore, using the arguments relating to some supposed physical configuration (e.g., the required property of the fields at infinity) to throw away a term from the Lagrangian is not advised. Besides, $F^{\prime} \tilde{F}^{\prime}$ may not be a complete divergence of some 4-vector. The suspicion emerges if we carefully compare Eqs. (2) and (4). As we know, $F$ and $\tilde{F}$ in Eq. (2) satisfy the following Maxwell equations:

$$
\partial_{\mu} F^{\mu \nu}=J^{\nu}, \quad \partial_{\mu} \tilde{F}^{\mu \nu}=0
$$

where $J_{\nu}$ is the electrically charged current, $J^{\nu}=\bar{\psi} \gamma^{\nu} \psi$. In order to have the second equation work (which cannot be obtained directly from the Lagrangian), the normal practice is 
to assume $A_{\mu}$ to be analytic. On the other hand, directly applying the Euler-Lagrangian principle to $\mathcal{L}^{\prime}$ we find the first Maxwell equation for $F^{\prime}$ to be

$$
\partial_{\mu} F^{\prime \mu \nu}-\theta \partial_{\mu} \tilde{F}^{\prime \mu \nu}=J^{\nu}
$$

In order to find the second equation for $F^{\prime}$, we must answer the question of whether we can also assume the analyticity of $A^{\prime} \mu$. If we assume so, we will have $\partial_{\mu} \tilde{F}^{\prime \mu \nu}=0$, and $F^{\prime \mu \nu}$ will satisfy the same equation as $F^{\mu \nu}$. However, we may as well assume

$$
\theta \partial_{\mu} F^{\prime \mu \nu}+\partial_{\mu} \tilde{F}^{\prime \mu \nu}=0
$$

instead. Indeed, if we regard $F$ and $\tilde{F}$ as two orthogonal vectors, then the "dual" rotated vectors $\theta F+\tilde{F}$ and $F-\theta \tilde{F}$ are also mutually orthogonal. Combining this with Eq. (7) we find that the Maxwell equations for $F^{\prime}$ and $\tilde{F}^{\prime}$ should be

$$
\partial_{\mu} F^{\prime \mu \nu}=J^{\nu} / \sqrt{1+\theta^{2}}, \quad \partial_{\mu} \tilde{F}^{\prime \mu \nu}=-\theta J^{\nu} / \sqrt{1+\theta^{2}}
$$

where $J^{\nu}$ is the same electrically charged current as in Eqs. (7). Note the differences between the Maxwell equations for Lagrangian Eq. (4), which has a $\theta$-term, and that for Lagrangian Eq. (2), which has no $\theta$-term. Both sets of the Maxwell equations can be inhomogeneous when the $\theta$-term is present. The second equation in Eqs. (8) means that $\partial_{\mu} \partial_{\nu} A_{\varrho}^{\prime} \neq \partial_{\nu} \partial_{\mu} A_{\varrho}^{\prime}$ at the source, so $F_{\mu \nu}^{\prime} \tilde{F}^{\prime} \mu \nu$ is not a complete divergence of a 4 -vector. Furthermore, the Green's function, which is the $A_{\mu}^{\prime}$ satisfying Eqs. (8) with a point source, is unknown due to the lack of mathematical tools to handle the equations. ${ }^{8}$ Anyway, we suspect that the $\theta$-term in QED may not be as simple as previously claimed. Putting all these considerations together, it becomes questionable whether one can do perturbative calculation with internal photons using Lagrangian $\mathcal{L}^{\prime}$.

In any case, it is worthwhile to check the no-effect theorem of FKW by a direct calculation starting from Eq. (2), even if the equivalence argument mildly criticized above is correct. We shall see that this calculation is much more involved conceptually.

The perturbation based on Lagrangian $\mathcal{L}$ works perfectly well. The free Dirac equation of the fermion with a complex mass is (in the momentum representation)

$$
(i \not p-\tilde{m}) \psi=0
$$


and the corresponding Klein-Gordon equation is $\left(p^{2}-m^{2}\right) \psi=0$. The propagator of the electron is

$$
\frac{1}{\not p-\tilde{m}+i \varepsilon}=\frac{\not p+\tilde{m}}{p^{2}-m^{2}+i \varepsilon} .
$$

The positive and negative energy projection operators are, respectively,

$$
\Sigma u_{h} \bar{u}_{h}=\tilde{m}^{\dagger} \frac{\not p+\tilde{m}}{2 m^{3}} \tilde{m}^{\dagger}, \Sigma v_{h} \bar{v}_{h}=-\tilde{m}^{\dagger} \frac{\not p-\tilde{m}}{2 m^{3}} \tilde{m}^{\dagger}
$$

as $\bar{u} \tilde{m} u=-\bar{v} \tilde{m} v=m$ and $\bar{u} \tilde{m} v=0$. The Dirac Eq. (9) is invariant under the following O-transformation:

$$
\psi(\vec{p}) \longrightarrow \psi^{\prime}(\vec{p})=\Omega \psi(-\vec{p})
$$

where $\Omega=\gamma_{o} e^{-i \gamma_{5} \phi}$. It seems that this transformation could take the role of the standard space reflection of the real mass QED. However, the fact that it involves a chiral transformation complicates things. The O-transformed Lagrangian will contain an extra " $\theta$-term." The O-invariance of the fermionic part, if assumed, requires $\left(\vec{A}, A_{o}\right)$ to go to $\left(-\vec{A}, A_{o}\right)$ under O-transformation, which fixes the O-transformation properties of $A_{\mu}$ and $F_{\mu \nu}$ and leaves no room to find a way to cancel the extra $\theta$-term. It is interesting to notice, however, that $\mathrm{O}$-invariance breaks only when there are triangle VVA or AAA diagrams, and in the standard QED perturbation calculations we never meet axial vertex. So $\mathrm{O}$-invariance can be a good perturbative symmetry. The following are the only operators which enjoy $\mathrm{O}$-invariance:

$$
\gamma_{\mu}, \tilde{m}, \tilde{m} \sigma_{\mu \nu}
$$

Note that two of the three operators appear in $\mathcal{L}$.

It is not surprising that in loop calculations only operators in Eq. (13) appear in fermion bilinears. Our concrete one-loop calculations show exactly this behavior. Therefore the phase parameter $\phi$ is non-renormalizable-that is, the QED with a complex mass needs exactly the same number of renormalization constants as the QED with a real fermion mass. The phase $\phi$ is convergent in loop calculations, although the imaginary part of the mass is not. Since $\theta$ is related to $\phi$ by the chiral rotation (3), it is also convergent. The first diagram which contributes the third operator in Eq. (13) is the one-loop proper vertex diagram which gives

$$
i \frac{\alpha}{4 \pi m^{2}} \bar{\psi} \tilde{m} \sigma_{\mu \nu} q^{\mu} A^{\nu} \psi
$$

The diagrams with only fermion loops and outgoing photons turn out always to be P-even. For example, the fermion loop with four outgoing photons can in principle have a term 
$a F^{2} F \tilde{F}$ which is $\mathrm{P}$-odd. But perturbative calculation gives $a=0$. The relevant part is

$$
\begin{gathered}
\operatorname{Tr} \gamma_{\mu}\left(\not p_{1}+\tilde{m}^{\dagger}\right) \gamma_{\nu}\left(\not \not_{2}+\tilde{m}^{\dagger}\right) \gamma_{\varrho}\left(\not \not_{3}+\tilde{m}^{\dagger}\right) \gamma_{\sigma}\left(\not \not_{4}+\tilde{m}^{\dagger}\right) \\
=\operatorname{Tr} \gamma_{\mu} \not \phi_{1} \gamma_{\nu} \not \not_{2} \gamma_{\varrho} \not \not_{3} \gamma_{\sigma} \not p_{4} \\
+\operatorname{Tr} \gamma_{\mu} \tilde{m}^{\dagger} \gamma_{\nu} \tilde{m}^{\dagger} \gamma_{\varrho} \not p_{3} \gamma_{\sigma} \not p_{4}+\ldots \\
=P-\text { even. }
\end{gathered}
$$

The first term is always $\mathrm{P}$-even in any regularization scheme. The second and following terms are convergent, so the relation

$$
\gamma_{\mu} \tilde{m}=\tilde{m}^{\dagger} \gamma_{\mu}
$$

can be used, leading to our conclusion. When there are only two outgoing photons in the fermion loop diagram, we choose the Pauli-Villars regularization to prove our conclusion. When calculating the process $\gamma^{*} \rightarrow e^{+} e^{-}$, we find no $\mathrm{P}$-violation effects such as longitudinal polarization of the electron with a complex mass. This is consistent with the conclusion that all fermion loops in QED are P-even, even when the fermion mass is complex. As we know, the sum over the final configuration is essentially putting the final fermion in a loop.

Now comes the sensitive problem of whether the electron with a complex mass has an electric dipole moment (EDM). The magnetic moment of the electron can be clearly identified only when the 4-component spinor wave function is split into two 2-component ones. The same is true for EDM. We should take a solution of Eq. (9) and substitute it into $e \bar{\psi} A \psi$ to look for the lowest order EDM, then substitute it into Eq. (14) for higher order corrections. There are at least two possible solutions to choose:

$$
\psi_{1}=\sqrt{\frac{E+m}{2 E}} e^{i \gamma_{5} \phi / 2}\left(\begin{array}{c}
\varphi \\
\frac{\vec{\sigma} \cdot \vec{p}}{E+m} \varphi
\end{array}\right) \equiv e^{i \gamma_{5} \phi / 2} u_{+},
$$

where $u_{+}$is the parity-even solution of the free Dirac equation with a real fermion mass; the other is the direct solution of Eq. (9):

$$
\psi_{2}=\sqrt{\frac{E+m_{1}}{2 E}}\left(\begin{array}{c}
\varphi \\
\frac{\vec{\sigma} \cdot \vec{p}+i m_{2}}{E+m_{1}} \varphi
\end{array}\right) .
$$

Since both solutions describe the free electron with the same complex mass, we are in general free to choose either of them to do perturbation. If we choose $\psi_{2}$ as the free 
electron wave function, we obtain

$$
E_{2}^{\prime}=\bar{\psi}_{2} e \not A \psi_{2}=\varphi^{\dagger}\left[e \Phi-\frac{e \vec{A} \cdot \vec{p}}{E}-\frac{e \vec{\sigma} \cdot \vec{B}}{2 E}-\frac{e \vec{\sigma} \cdot \vec{E} \times \vec{p}}{2 E\left(E+m_{1}\right)}+\frac{i e \vec{E} \cdot \vec{p}}{2 E\left(E+m_{1}\right)}-\frac{e m_{2} \vec{\sigma} \cdot \vec{E}}{2 E\left(E+m_{1}\right)}\right] \varphi
$$

The character of this perturbative energy is that it has a P-odd term, the EDM term (the last one). In the non-relativistic limit, the EDM in Eq. (17) is $\frac{e}{2 m} \tan (\phi / 2)$. We can be encouraged about this result by the corresponding result obtained through the Foldy-Wouthuysen transformation. We also find higher order corrections to the EDM in calculation of the relevant loop diagrams. However, if we take $\psi_{1}$ as the free electron wave function, we obtain, instead of Eq. (17),

$$
E_{1}^{\prime}=\bar{\psi}_{1} e \not A \psi_{1}=\bar{u}_{+} e \not A u_{+}=\varphi\left[e \Phi-\frac{e \vec{A} \cdot \vec{p}}{E}-\frac{e \vec{\sigma} \cdot \vec{B}}{2 E}-\frac{e \vec{\sigma} \cdot \vec{E} \times \vec{p}}{2 E(E+m)}+\frac{i e \vec{E} \cdot \vec{p}}{2 E(E+m)}\right] \varphi
$$

which has no EDM term. (Note Eqs. (17) and (18) have the first three terms in common.) Furthermore, the information of the complex phase $\phi$ also disappears in all higher order corrections. Indeed, as discussed before, the most gene:al form of the higher order vertex corrections in the QED with a complex fermion mass is

$$
\bar{\psi}\left[V_{\mu} \gamma^{\mu}+\tilde{m}\left(S+T_{\mu \nu} \sigma^{\mu \nu}\right)\right] \psi
$$

where $S, V_{\mu}$, and $T_{\mu \nu}$ are scalar, vector, and tensor functions, respectively, of $A_{\mu}$ and $q_{\mu}$ (the 4-momentum of the photon). When we take $\psi$ as $\psi_{1}$ in Eq. (15), Eq. (19) becomes

$$
\bar{u}_{+}\left[V_{\mu} \gamma^{\mu}+m\left(S+T_{\mu \nu} \sigma^{\mu \nu}\right)\right] u_{+}
$$

which has no trace of the phase. The most general solutions of the free Dirac equation and the problem of which free wave function should be chosen to do perturbation are discussed in detail by the authors with Gupta. ${ }^{9}$ By a comparison with the energy of the relativistic electron (with a complex mass) in the Coulomb potential, which is rigorously solvable, we conclude that Eq. (15) should be the correct free-wave function to use in perturbation. Indeed, the resulting energy of the electron in the Coulomb potential does not know the phase of its complex mass, even if weak external electric field is added. It is unreasonable to assume that it becomes sensitive to the phase as in Eq. (18) only because the electron is moved from the Coulomb potential (plus perturbation) to some other one and perturbation based on free Dirac equation solutions must apply. Therefore, the phase of the complex fermion mass defined in Eqs. (1) and (2) is not observable in QED, as shown in Eq. (20). 
In conclusion, the complex mass in Lagrangian Eq. (2) provides exactly the same physics as when the phase of the mass vanishes, if perturbation is applied-that is, the phase $\phi$ is a redundant parameter. We would like to make a final remark. Our conclusions about nil EDM will not change when $\tilde{m}$ in Eq. (2) is replaced by $\tilde{m}\left(1+\sigma+i \gamma_{5} \pi^{o}\right)$ with $\sigma$ and $\pi^{\circ}$, scalar and pseudoscalar fields, respectively, and the corresponding parity-conserved Lagrangian for $\sigma$ and $\pi^{o}$ fields is added to Eq. (2). 


\section{ACKNOWLEDGEMENTS}

The authors would like to thank Z. Huang and X.G. He for useful discussions. Authors

in Ref. 10 are acknowledged for pointing out to Wu that the symmetry of Eq. (12) is observed in their preprint. 


\section{REFERENCES}

1. G. Feinberg, P.K. Kabir, and S. Weinberg, Phys. Rev. Lett. 3, 111; 224(E) (1959).

2. R. Dashen, Phys. Rev. D 3, 1879 (1971). J. Nuyts, Phys. Rev. Lett. 26, 1604 (1971).

3. R.D. Peccei and H.R. Quinn, Phys. Rev. Lett. 38, 1440; Phys. Rev. D 116, 1791 (1977).

4. V. Baluni, Phys. Rev. D 19, 2227 (1979). R. Crewther, in Field Theoretical Methods in Particle Physics, NATO Advanced Study Institute, Kaiseslautern, Germany, 1979, ed. W. Ruhl (Plenum Press, New York and London, 1980); P. Di Vecchia and G. Veneziano, Nucl. Phys, B171, 253 (1980); E. Witten, Ann. Phys. (NY) 128, 363 (1980); G. 't Hooft, Phys. Rep. 142, 357 (1986).

5. H. Banerjee, D. Chatterjee and P. Mitra, Saha Institute preprint, SINP-TNP-90/17; R.G. Sachs, Enrico Fermi Institute preprint; A. Abada, J. Galand, A. Le Yaouanc, L. Oliver, and O. Pene, Phys. Lett. 256B, 508 (1991).

6. Z. Huang, K. Vishwanathan, and D.D. Wu, Simon Fraser University preprint, July-90-1, unpublished; and Mod. Phys. Lett. A8, 711 (1991).

7. J. Steinberger, Phys. Rev. 76, 1180 (1949); S. Adler, Phys. Rev. 177, 2426 (1969); J. Bell and R. Jackiw, Nov. Cim. 60, 47 (1969); W.A. Bardeen, Phys. Rev. 184, 1848 (1969); J. Wess and B. Zumino, Phys. Lett. 37B, 95 (1971); K. Fujikawa, Phys. Rev. Lett. 42, 1195 (1979) and Phys. Rev. D 21, 2848 (1980).

8. For early discussions of such equations, see P.A.M. Dirac, Proc. R. Soc. London A133, 60 (1931); Phys. Rev. 74, 817 (1948); A. Goldhaber, J. Schwinger, ibid., 144, 1087 (1966); Phys. Rev. D 12, 3105 (1975); D. Zwanziger, Phys. Rev. 176, 1489 (1968); T.T. Wu and C.N. Yang, Nucl. Phys. B107, 365 (1976); R.A. Brandt, F. Nari, and D. Zwanziger, Phys. Rev. Lett. 40, 147 (1978).

9. V. Gurta, B.J. McKellar, and D.D. Wu, University of Melbourne preprint, UM-P$91 / 49$.

10. H. Banerjee, D. Chatterjee, and P. Mitra, Saha Institute Preprint, SINP-TNP-90/5. 


\section{Disclaimer Notice}

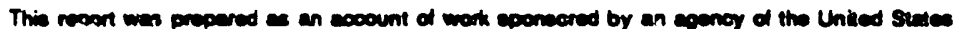
Covernmen. Neither the United Stave Cowarnment of any epency thereet. ner eny of their

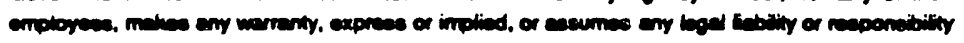

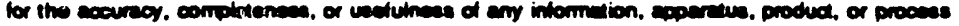

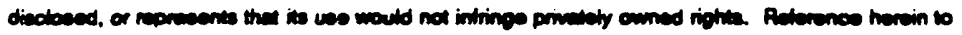

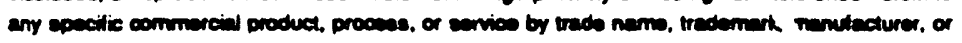

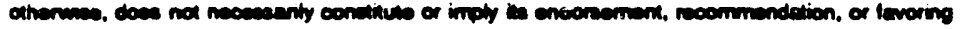

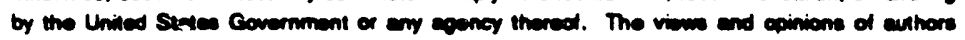

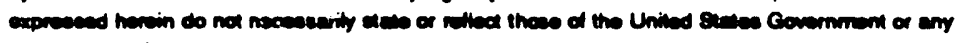
anoncy thereat.

Superconducting Super Collider Laboratory is an equal opportunity employer. 

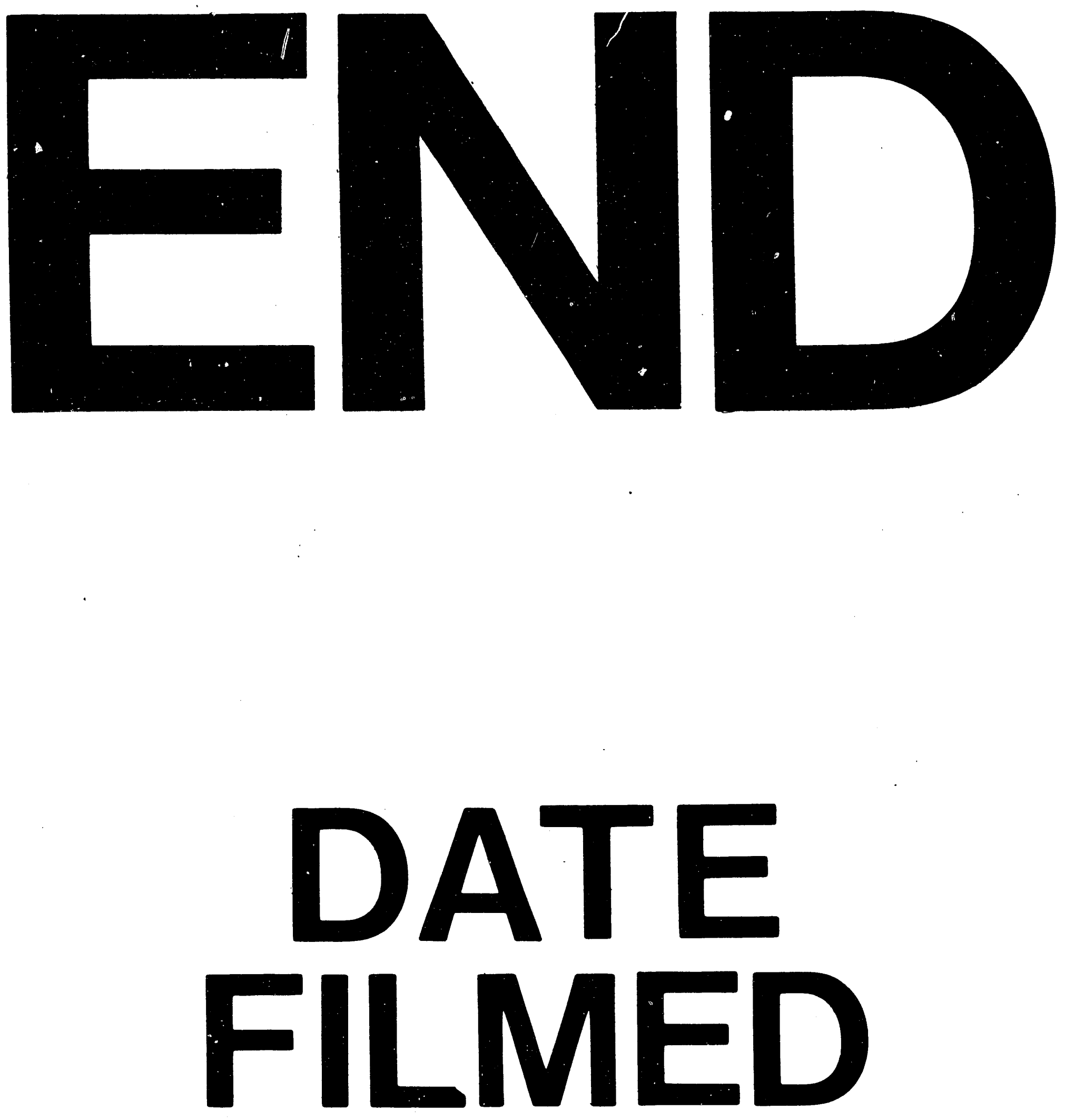

1

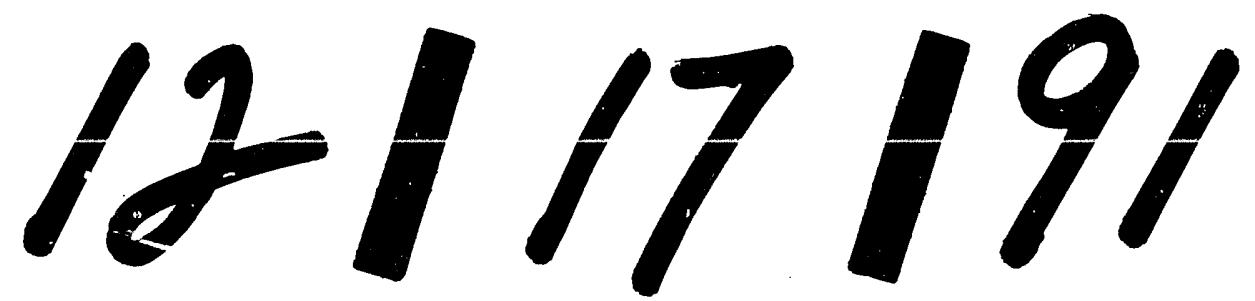


